\title{
APPROXIMATION METHODS AND THE GENERALISED FULLER INDEX FOR SEMI-FLOWS IN BANACH SPACES
}

\author{
by A. J. B. POTTER \\ (Received 11th September 1984)
}

\section{Introduction}

In [3] Fuller introduced an index (now called the Fuller index) in order to study periodic solutions of ordinary differential equations. The objective of this paper is to give a simple generalisation of the Fuller index which can be used to study periodic points of flows in Banach spaces. We do not claim any significant breakthrough but merely suggest that the simplistic approach, presented here, might prove useful for the study of non-linear differential equations. We show our results can be used to study functional differential equations.

Our techniques are based on those of Browder and Petryshyn [1], the index we define in this paper generalises the Fuller index in much the same way that the $A$-proper degree theory extends Brouwer degree theory. There are obvious extensions of our work but in order to minimise technicalities we choose to omit these and concentrate on simple cases.

The results in this paper were announced in [5].

\section{Preliminaries}

In this paper we use the Fuller index for flows in finite dimensional spaces. We recall the basic properties below.

Consider the ordinary differential equation

$$
\dot{y}=f(y), \quad y \in Y
$$

where $Y$ is a finite dimensional vector space and $f$ is a locally Lipschitzean mapping from $Y$ into $Y$. Let $T(t, a)$ denote the associated flow with initial point $a$. The set $\Pi(f)$ of periodic points of $(2.1)$ is defined by

$$
\Pi(f)=\{(p, a) \in[0, \infty) \times Y: T(p, a)=a\} .
$$

Fix a bounded open subset $\Omega \subset(0, \infty) \times Y$ which is bounded away from $\{0\} \times Y$. Assume that there are no periodic points intersecting the boundary $\partial \Omega$ of $\Omega$, that is

$$
\Pi(f) \cap \partial \Omega=\phi
$$


(so in particular no fixed points of (2.1) can lie in the projection of $\Omega$ on $Y$ ). Then the Fuller index, $i(f, \Omega)$, is a rational number which has the following properties (see [3], [2]).

(P1) (Existence) If $i(f, \Omega) \neq 0$ then $\Pi(f) \cap \Omega \neq \phi$.

(P2) (Additivity) If $\Omega=\Omega_{1} \cup \Omega_{2}$ where $\Omega_{1}, \Omega_{2}$ are open subsets of $Y$ and $\Pi(f) \cap$ $\left\{\left(\Omega_{1} \cap \Omega_{2}\right) \cup \partial \Omega_{1} \cup \partial \Omega_{2}\right\}=\phi$ then

$$
i(f, \Omega)=i\left(f, \Omega_{1}\right)+i\left(f, \Omega_{2}\right) .
$$

(P3) (Homotopy Invariance) If $\left\{f^{\alpha}: 0 \leqq \alpha \leqq 1\right\}$ is a homotopy of vector fields (i.e. the map $\alpha \mapsto f^{\alpha}$ is a continuous map from [0,1] into $C(Y, Y)$ (compact-open topology) with $f^{\alpha}$ locally Lipschitzean for each $\left.\alpha \in[0,1]\right)$ and $\Pi\left(f^{\alpha}\right) \cap \partial \Omega=\phi$ for each $\alpha \in[0,1]$, then $i\left(f^{\alpha}, \Omega\right)$ is independent of $\alpha$.

Let $X$ be a Banach space, we denote the norm on $X$ by $\|\cdot\|$. For a subset $S$ of $X, \partial S$ and $\bar{S}$ denote the boundary and closure of $S$ in $X$. We need the following concepts.

Definition 2.1. An approximation scheme for $X$ is a sequence $\left\{Y_{n}, p_{n}, q_{n}\right\}$ where for each integer $n \geqq 0, Y_{n}$ is a finite dimensional vector space, $p_{n}$ and $q_{n}$ are continuous maps with $p_{n}$ mapping $X$ into $Y_{n}, q_{n}$ mapping $Y_{n}$ into $X$ and such that $q_{n}^{-1}(B)$ is bounded for all bounded subsets $B$ of $X$.

Let $\left\{Y_{n}, p_{n}, q_{n}\right\}$ be an approximation scheme for $X$ and let $\Omega$ be a bounded open subset of $[0, \infty) \times X$. For each integer $n$, let

$$
\Omega_{n}=\left\{(t, y) \in[0, \infty) \times Y_{n}:\left(t, q_{n}(y)\right) \in \Omega\right\} .
$$

Then $\Omega_{n}$ is a bounded open subset of $[0, \infty) \times Y_{n}$ and we note that $Q_{n}\left(\bar{\Omega}_{n}\right) \subset \bar{\Omega}$ and $Q_{n}\left(\partial \Omega_{n}\right) \subset \partial \Omega$ where $Q_{n}(t, y)=\left(t, q_{n}(y)\right)$. For examples of approximation schemes see [1].

Definition 2.2. A $C^{0}$-semiflow (flow) in $X$ is a continuous mapping $T$ from an open subset of $D$ of $[0, \infty) \times X(\mathbb{R} \times X)$ into $X$ which has the following properties

(i) $T(0, x)=x$,

(ii) $T(s+t, x)=T(s, T(t, x))$

(it being understood that these properties hold whenever they make sense; in fact we always assume $D$ has the following properties

(a) $(0, x) \in D$ for all $x \in X$,

(b) if $(t, x) \in D$ and $(s, T(t, x)) \in D$ then $(s+t, x) \in D)$.

Let $\left\{Y_{n}, p_{n}, q_{n}\right\}$ be an approximation scheme for $X$ and let $T$ be a $C^{0}$-semiflow on $X$. For each integer $n \geqq 0$ we define the maps $f_{n}$ by

$$
f_{n}(y)=\lim _{t \rightarrow 0_{+}} p_{n}\left(\left(T\left(t, q_{n}(y)\right)-q_{n}(y)\right) / t\right)
$$

the domain of $f_{n}$ being those $y \in Y_{n}$ for which the limit exists. 
Definition 2.3. A $C^{0}$-semiflow $T$ in $X$ is said to be approximable with respect to the approximation scheme $\left\{Y_{n}, p_{n}, q_{n}\right\}$ if for each $n \geqq 0$ the domain of $f_{n}$ is $Y_{n}$ and $f_{n}: Y_{n} \rightarrow Y_{n}$ is a locally Lipschitzean mapping.

If a $C^{0}$-semiflow $T$ is approximable with respect to an approximation scheme $\left\{Y_{n}, p_{n}, q_{n}\right\}$ then for each $n$ there is an associated $C^{0}$-flow in $Y_{n}$, namely the flow generated by the ordinary differential equation

$$
\dot{y}=f_{n}(y), \quad y \in Y_{n} .
$$

We denote these flows by $T_{n}$. For many. semiflows $T_{n}$ is a "good" approximation to $T$ in the sense of the next definition.

Definition 2.4. A $C^{0}$-semiflow $T$ in $X$ is said to be $F$-proper (with respect to the approximation scheme $\left.\left\{Y_{n}, p_{n}, q_{n}\right\}\right)$ if $T$ is approximable and for any sequence $\left\{n_{j}\right\}$ of integers with $n_{j} \rightarrow \infty$ and corresponding bounded sequence $\left\{\left(t_{n_{j}}, x_{n_{j}}\right)\right\}$ in $[0, \infty) \times X$ with $x_{n_{j}}=q_{n_{j}}\left(y_{n_{j}}\right)$ for some $y_{n_{j}} \in Y_{n_{j}},\left\{t_{n_{j}}\right\}$ bounded away from zero and such that $y_{n_{j}}=$ $T_{n_{j}}\left(t_{n_{j}}, y_{n_{j}}\right)$ then there is a subsequence $\left\{n_{j(k)}\right\}$ such that $t_{n_{j(k)}} \rightarrow t, x_{n_{j(k)}} \rightarrow X$ and $x=$ $T(t, x)$.

The concept of an $F$-proper semiflow is motivated by the definition of $A$-proper (see [1]). There are several examples of $F$-proper semiflows.

\section{The generalised Fuller index}

In this section we define the generalised Fuller index and discuss the properties of this index. Throughout this section $X$ denotes a Banach space with an approximation scheme $\left\{Y_{n}, p_{n}, q_{n}\right\}$ and $T$ denotes a $C^{0}$-semiflow on $X$. The set $\Pi(T)$ of periodic points of $T$ is defined by

$$
\Pi(T)=\{(\tau, x) \in[0, \infty) \times X: x=T(\tau, x)\}
$$

We consider a bounded open subset $\Omega$ of $[0, \infty) \times X$ such that $\Omega$ is bounded away from $\{0\} \times X$. We use the same notation as in the previous section.

Lemma 3.1 Suppose that $T$ is F-proper with respect to the approximation scheme $\left\{Y_{n}, p_{n}, q_{n}\right\}$ and suppose $\Pi(T) \cap \partial \Omega=\phi$. Then there exists an integer $N$ such that $\Pi\left(f_{n}\right) \cap \partial \Omega_{n}=\phi$ for all $n \geqq N$.

Proof. Suppose the assertion of the lemma is false. Then there exists a sequence $\left\{\boldsymbol{n}_{j}\right\}$ of integers with $n_{j} \rightarrow \infty$ and a sequence $\left\{\left(t_{n_{j}}, x_{n_{j}}\right)\right\}$ with $x_{n_{j}}=q_{n_{j}}\left(y_{n_{j}}\right)$ where $\left(t_{n_{j}}, y_{n_{j}}\right) \in \partial \Omega_{n_{j}}$ and $y_{n_{j}}=T_{n_{j}}\left(t_{n_{j}}, y_{n_{j}}\right)$. We note that $\left(t_{n_{j}}, x_{n_{j}}\right) \in \partial \Omega$ and as $\partial \Omega$ is bounded and bounded away from $\{0\} \times X$, it follows from the $F$-properness of $T$ that we can find a subsequence $\left\{\left(t_{n_{j(k)},}, x_{n_{j(k)}}\right)\right\}$ such that $t_{n_{j(k)}} \rightarrow t>0, x_{n_{j(k)}} \rightarrow x$ and $x=T(t, x)$. But $\partial \Omega$ is closed so $(t, x) \in \partial \Omega$ which contradicts our hypothesis.

The lemma shows that $i\left(f_{n}, \Omega_{n}\right)$ is defined for $n \geqq N$. This fact allows the following definition. 
Definition 3.2. Suppose that $\Pi(T) \cap \partial \Omega=\phi$. Then we define $I(T, \Omega)=\left\{\gamma \in \mathbb{R}^{*}\right.$ : there is an infinite sequence $\left\{n_{j}\right\}$ of non-negative integers with $n_{j} \rightarrow \infty$ such that $\left.i\left(f_{n}, \Omega_{n_{j}}\right) \rightarrow \gamma\right\}$ (here $\mathbb{R}^{*}=\mathbb{R} \cup\{\infty\} \cup\{-\infty\}$ ). We call $I(T, \Omega)$ the generalised Fuller index of $T$. (Note that $I(T, \Omega)$ is closed.)

Although $I(T, \Omega)$ is possibly a set with many elements, it has properties similar to those of the Fuller index.

Theorem 3.3. Suppose that $T$ is $F$-proper with respect to the approximation scheme $\left\{Y_{n}, p_{n}, q_{n}\right\}$ and suppose that $\Pi(T) \cap \partial \Omega=\phi$. Then $I(T, \Omega)$ is a non-empty subset of $\mathbb{R}^{*}$ for which the following assertions hold.

(i) (Existence) If $I(T, \Omega) \neq\{0\}$ then $\Pi(T) \cap \Omega \neq \phi$.

(ii) (Additivity) Let $\Omega=\Omega_{1} \cup \Omega_{2}$ and suppose that $\Pi(T) \cap\left\{\left(\Omega_{1} \cup \Omega_{2}\right) \cup \partial \Omega_{1} \cup \partial \Omega_{2}\right\}=\phi$. Then

$$
I(T, \Omega) \subseteq I\left(T, \Omega_{1}\right)+I\left(T, \Omega_{2}\right)
$$

with equality holding if either $I\left(T, \Omega_{1}\right)$ or $I\left(T, \Omega_{2}\right)$ is a singleton (where for two subsets $I_{1}$ and $I_{2}$ of $\mathbb{R}^{*}$ we set $I_{1}+I_{2}=\left\{\gamma: \gamma=\gamma_{1}+\gamma_{2}\right.$ with $\left.\gamma_{1} \in I_{1}, \gamma_{2} \in I_{2}\right\}$ and apply the convention that $\infty+(-\infty)=\gamma$ for every $\left.\gamma \in \mathbb{R}^{*}\right)$.

Proof. As remarked previously the fact that $I(T, \Omega)$ is well defined follows from Lemma 3.1. We prove (i). Suppose that $I(T, \Omega) \neq 0$. Then there exists a sequence $\left\{n_{j}\right\}$ of integers with $n_{j} \rightarrow \infty$ such that $i\left(f_{n_{j}}, \Omega_{n_{j}}\right) \rightarrow p$ where $p \in I(T, \Omega), p \neq 0$. In particular we can find sequences $\left\{t_{n_{j}}\right\},\left\{y_{n_{j}}\right\}$ with $\left(t_{n_{j}}, y_{n_{j}}\right) \in \Omega_{n_{j}}$ and $y_{n_{j}}=T_{n_{j}}\left(t_{n_{j}}, y_{n_{j}}\right)$ (this follows from property (P1) of the Fuller index). Put $x_{n_{j}}=q_{n_{j}}\left(y_{n_{j}}\right)$. Since $T$ is $F$-proper, we may assume (passing to a subsequence if necessary) that $x_{n_{j}} \rightarrow x, t_{n_{j}} \rightarrow t$ and $x=T(t, x)$. Since $\left(t_{n_{j}}, x_{n_{j}}\right) \in \Omega,(t, x) \in \bar{\Omega}$ but $\Pi(T) \cap \partial \Omega=\phi$ so $(t, x) \in \Omega$. Hence $(t, x) \in \Pi(T) \cap \Omega$. The proof of (ii) follows from property (P2) of the Fuller index.

Definition 3.4. We say $\left\{T^{\alpha}: 0 \leqq \alpha \leqq 1\right\}$ is an $F$-proper homotopy of semiflows in $X$ with respect to an approximation scheme $\left\{Y_{n}, p_{n}, q_{n}\right\}$ if and only if for each $\alpha \in[0,1], T^{\alpha}$ is approximable, $\left\{f_{n}^{\alpha}: 0 \leqq \alpha \leqq 1\right\}$ is a homotopy of vector fields on $Y_{n}$ for each $n$ and for any sequence $\left\{n_{j}\right\}$ with $n_{j} \rightarrow \infty$ and corresponding bounded sequence $\left\{\left(\alpha_{n_{j}}, t_{n_{j}}, x_{n_{j}}\right)\right\}$ in $[0,1] \times[0, \infty) \times X$ with $x_{n_{j}}=q_{n_{j}}\left(y_{n_{j}}\right)$ for some $y_{n_{j}} \in Y_{n_{j}},\left\{t_{n_{j}}\right\}$ bounded away from zero and such that $y_{n_{j}}=T_{n_{j}}^{\alpha_{n_{j}}}\left(t_{n_{j}}, y_{n_{j}}\right)$ then there is a subsequence $\left\{n_{j(k)}\right\}$ such that $\alpha_{n_{j(k)}} \rightarrow \alpha, t_{n_{j(k)}} \rightarrow t, x_{n_{j(k)}} \rightarrow x$ and $x=T^{\alpha}(t, x)$.

Theorem 3.5. Let $\left\{T^{\alpha}: 0 \leqq \alpha \leqq 1\right\}$ be an F-proper homotopy of semiflows in $X$ with respect to the approximation scheme $\left\{Y_{n}, p_{n}, q_{n}\right\}$ and suppose that $\Pi\left(T^{\alpha}\right) \cap \partial \Omega=\phi$ for $0 \leqq \alpha \leqq 1$. Then $I\left(T^{\alpha}, \Omega\right)$ is independent of $\alpha$.

Proof. It is sufficient to show that there exists an integer $N$ such that $\Pi\left(T_{n}^{\alpha}\right) \cap \partial \Omega_{n}=\phi$ for all $n \geqq N$ and $0 \leqq \alpha \leqq 1$. (For then the homotopy property (P3) of the Fuller index implies that $i\left(f_{n}^{\alpha}, \Omega_{n}\right)$ is independent of $\alpha$ from which our result follows.)

Suppose, for contradiction, this is not the case. Then there is a sequence $\left\{n_{j}\right\}$ of 
integers with $n_{j} \rightarrow \infty$ and corresponding sequences $\left\{\alpha_{n_{j}}\right\},\left\{t_{n_{j}}\right\},\left\{y_{n_{j}}\right\}$ where $0 \leqq x_{n_{j}} \leqq 1$,

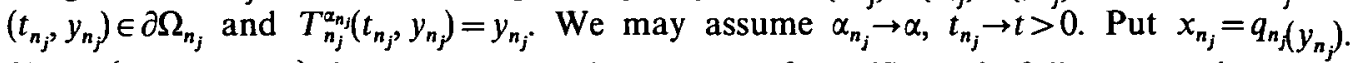
Since $\left\{T^{\alpha}: 0 \leqq \alpha \leqq 1\right\}$ is an $F$-proper homotopy of semiflows it follows, passing to a subsequence, if necessary, that $x_{n_{j}} \rightarrow x$ and $x=T^{\alpha}(t, x)$. Since $\left(t_{n_{j}}, y_{n_{j}}\right) \in \partial \Omega_{n_{j}},\left(t_{n_{j}}, x_{n_{j}}\right) \in \partial \Omega$ so $(t, x) \in \partial \Omega$ and thus $\Pi\left(T^{\alpha}\right) \cap \partial \Omega \neq \phi$. Contradiction.

\section{Functional differential equations}

In this section we show that the semiflows generated by functional differential equations are $F$-proper with respect to a certain approximation scheme.

For $r>0$ let $X=C([-r, 0], \mathbb{R})$ be the space of continuous functions from $[-r, 0]$ to $\mathbb{R}$; endowed with the norm $\|\phi\|=\sup \{|\phi(\theta)|:-r \leqq \theta \leqq 0\}, X$ becomes a Banach space. For $x \in C([-r, A], \mathbb{R}), A>0$ let $x_{t} \in X$ for $t \in[0, A]$ be defined by $x_{t}(\theta)=x(t+\theta)$, $-r \leqq \theta \leqq 0$. We consider the functional differential equation

$$
\dot{x}(t)=f\left(x_{t}\right)
$$

where we assume

(H1) $f: X \rightarrow \mathbb{R}$ is continuously Fréchet differentiable and $f^{\prime}$ maps bounded sets of $X$ into bounded sets of $X^{*}$,

(H2) there exists constants $A, B>0$ such that

$$
|f(x)| \leqq A+B\|x\| \text { for } x \in X .
$$

We note that (H1) implies $f$ is locally Lipschitzean and so (4.1) generates a $C^{0}$-semiflow $T$ in $X$. (If $x(t)=T(t, \phi)$ then $x(t)$ is the unique solution of (4.1) satisfying $x_{0}=\phi$.) The growth condition $(\mathrm{H} 2)$ guarantees that the domain of $T$ is $[0, \infty) \times X$ and $T$ maps bounded sets of $[0, \infty) \times X$ into bounded sets. We remark that $(\mathrm{H} 2)$ could be considerably weakened but it makes our calculations easier. (H1) could also be weakened at the expense of extra technicalities.

Let $D=\left[t_{0}, t_{1}, \ldots, t_{n}\right]$ be a dissection of $[-r, 0]$ so that $-r=t_{0}<t_{1}<t_{2}<\cdots<t_{n}=0$. Put $\Delta_{D, j}=t_{j+1}-t_{j}, 0 \leqq j<n$ and put $\Delta_{D}=\max \Delta_{D, j}$. Define $p_{D}: X \rightarrow \mathbb{R}^{n+1}, q_{D}: \mathbb{R}^{n+1} \rightarrow X$ by

$$
p_{D}(\phi)=\left(\phi\left(t_{0}\right), \phi\left(t_{1}\right), \ldots, \phi\left(t_{n}\right)\right) \text { for } \phi \in X
$$

and

$$
q_{D}(y)(s)=\left(\frac{s-t_{j}}{t_{j+1}-t_{j}}\right) y_{j+1}+\left(\frac{t_{j+1}-s}{t_{j+1}-t_{j}}\right) y_{j} \quad\left(t_{j} \leqq s \leqq t_{j+1}, 0 \leqq j<n\right)
$$

where $y=\left(y_{0}, y_{1}, y_{2}, \ldots, y_{n}\right) \in \mathbb{R}^{n+1}$. We use $|\cdot|$ to denote the sup-norm on $\mathbb{R}^{n+1}$. Note that $p_{D}, q_{D}$ are bounded linear operators, $\left\|p_{D}\right\| \leqq 1,\left\|q_{D}\right\|=1$ and $q_{D}^{-1}(B)$ is bounded for all bounded subsets $B$ of $X$.

Let $f_{D}: \mathbb{R}^{n+1} \rightarrow \mathbb{R}^{n+1}$ be defined by

$$
f_{D}(y)=\lim _{t \rightarrow 0_{+}} p_{D}\left(\left(T\left(t, q_{D}(y)\right)-q_{D}(y)\right) / t\right)
$$


where $T$ is the $C^{0}$-semiflow generated by (4.1). We note that $f_{D}$ is well defined; indeed, it is easy to show that

$$
f_{D}(y)_{j}= \begin{cases}\left(y_{j+1}-y_{j}\right) / \Delta_{D, j} & 0 \leqq j<n \\ f\left(q_{D}(y)\right) & j=n\end{cases}
$$

(where we use the notation $z_{j}$ for the $j$ th-component of $z \in \mathbb{R}^{n+1}$ ). Note also $f_{D} \in C^{1}\left(\mathbb{R}^{n+1}, \mathbb{R}^{n+1}\right)$ and is thus locally Lipschitzean.

Before we define precisely the approximation scheme we are to use, we consider properties of the flows $T_{D}$ in $\mathbb{R}^{n+1}$ generated by the ordinary differential equations

$$
\dot{y}=f_{D}(y), \quad y \in \mathbb{R}^{n+1}
$$

for dissections $D$ of $[-r, 0]$.

For notational convenience we write $\Delta_{j}$ for $\Delta_{D, j}$ and $\Delta$ for $\Delta_{D}$ in future.

Lemma 4.1. Let $M, P>0$ be constants and suppose that $y(t)$ is a solution of (4.4) with $|y(0)| \leqq M$. Then there is a constant $K>0$ (independent of $D$ ) such that

$$
|y(t)| \leqq K \quad \text { for all } 0 \leqq t \leqq P .
$$

Proof. Let $y(t)=\left(y_{0}(t), y_{1}(t), \ldots, y_{n}(t)\right)$ be a solution of (4.2) with $|y(0)| \leqq M$. Put $u(t)=|y(t)|$ so that $u(t)=\sup \left\{\left|y_{j}(t)\right|: 0 \leqq j \leqq n\right\}$. Let $G=\left\{t>0:\left|y_{n}(t)\right|<u(t)\right\}$. Thus for $t \in G$, $u(t)= \pm y_{j}(t)$ where $0 \leqq j<n$. Note that for such $j, \dot{y}_{j}(t)=\left(y_{j+1}(t)-y_{j}(t)\right) / \Delta_{j}$, so if $u(t)=$ $+y_{j}(t)$ then $\dot{y}_{j}(t) \leqq 0$, if $u(t)=-y_{j}(t)$ then $\dot{y}_{j}(t) \geqq 0$. From this observation we deduce that $u(t)$ is decreasing on every connected component of $G$. For simplicity, assume $G=$ $\bigcup_{r=1}^{R}\left(s_{2 r-1}, s_{2 r}\right)$ where $s_{1}>0$. Put $s_{0}=0$ then for $t \in\left[s_{2 r}, s_{2 r+1}\right]$ since $u(t)=\left|y_{n}(t)\right|$ and

$$
y_{n}(t)=y_{n}\left(s_{2 r}\right)+\int_{s_{2 r}}^{t} \dot{y}_{n}(s) d s
$$

it follows

$$
u(t) \leqq u\left(s_{2 r}\right)+\int_{s_{2 r}}^{t} \mid f\left(q_{D}(y(s)) \mid d s\right.
$$

Using the growth condition (H2) and the fact that $\left\|q_{D}(y(s))\right\| \leqq u(s)$ we obtain

$$
\begin{aligned}
u(t) & \leqq u\left(s_{2 r}\right)+\int_{s_{2 r}}^{t}(A+B u(s)) d s \leqq u\left(s_{2 r-1}\right)+\int_{s_{2 r}}^{t}(A+B u(s)) d s \\
& \leqq u\left(s_{2(r-1)}\right)+\int_{s_{2(r-1)}}^{s_{2 r-1}}(A+B u(s)) d s+\int_{s_{2 r}}^{t}(A+B u(s)) d s \\
& \leqq \ldots \text { etc. }
\end{aligned}
$$


And so, repeating the above calculations, we get

$$
u(t) \leqq u(0)+\int_{0}^{t}(A+B u(s)) d s
$$

This inequality holds for all $t \in[0, p]$ and so Gronwall's Inequality gives

$$
u(t) \leqq(u(0)+A P) e^{B P} \text { for } 0 \leqq T \leqq P .
$$

Put $K=(M+A B) e^{B P}$. A similar, but more complicated analysis applies when $G$ is a countable union of open intervals. This is left as an exercise for the reader.

Lemma 4.2. Let $M>0$ and $P>0$. Suppose that $y(t)$ is a periodic solution of (4.4) with $|y(0)| \leqq M$ and with minimal period $p<P$. Then there exist constants $L, N>0$ (independent of $D$ ) such that

$$
|\dot{y}(t)| \leqq L \text { and }|\ddot{y}(t)| \leqq N \quad \text { for all } t \in \mathbb{R}
$$

Proof. By Lemma 4.1 there exists $K>0$ such that $|y(t)| \leqq K$ for all $t \in \mathbb{R}$ (note $y$ is periodic). Put $z_{j}=\dot{y}_{j}$ and note that

$$
\dot{z}_{j}=\left(z_{j+1}-z_{j}\right) / \Delta_{j} \text { for } 0 \leqq j<n
$$

Put $k=1 / \Delta_{j}$ then

$$
\dot{z}_{j}+k z_{j}=k z_{j+1}
$$

Multiply this equation by $e^{k t}$ and integrate to obtain

$$
z_{j}(t)=e^{-k t} z_{j}(0)+e^{-k t} \int_{0}^{t} k z_{j+1}(s) e^{k s} d s
$$

As $z_{j}$ is periodic with period $p$, for all integers $m$

$$
\begin{aligned}
\left|z_{j}(t)\right| & =\left|z_{j}(t+m p)\right| \leqq e^{-k(t+m p)}\left|z_{j}(0)\right|+e^{-k(t+m p)} \int_{0}^{t+m p} k\left|z_{j+1}(s)\right| e^{k s} d s \\
& \leqq e^{-k(t+m p)}\left|z_{j}(0)\right|+\left(\sup _{t \in \mathbf{R}}\left|z_{j+1}(t)\right|\right)\left(1-e^{-k(t+m p)}\right) .
\end{aligned}
$$

Let $m \rightarrow \infty$ to obtain

$$
\sup _{t \in \mathbf{R}}\left|z_{j}(t)\right| \leqq \sup _{t \in \mathbb{R}}\left|z_{j+1}(t)\right| \text { for } 0 \leqq j<n
$$

Hence

$$
\sup _{t \in \mathbf{R}}|\dot{y}(t)|=\sup _{t \in \mathbf{R}} \sup _{0 \leqq j \leqq n}\left|\dot{y}_{j}(t)\right|=\sup _{0 \leqq j \leqq n} \sup _{t \in \mathbf{R}}\left|\dot{y}_{j}(t)\right|=\sup _{t \in \mathbf{R}}\left|\dot{y}_{n}(t)\right|
$$


By (H2), $\left|\dot{y}_{n}(t)\right|=\left|f\left(q_{D}(y(t))\right)\right| \leqq A+B\left|q_{D}(y(t))\right| \leqq A+B|y(t)|$, and so

$$
\left|\dot{y}_{n}(t)\right| \leqq A+B K \quad \text { for all } t \in \mathbb{R} .
$$

Put $L=A+B K$ to get $|\dot{y}(t)| \leqq L$ for all $t \in \mathbb{R}$.

Next put $w_{j}=\ddot{y}_{j}$ and noting $\dot{w}_{j}=\left(w_{j+1}-w_{j}\right) / \Delta_{j}$ for $0 \leqq j<n$ we conclude as before

$$
\sup _{t \in \mathbf{R}}\left|w_{j}(t)\right| \leqq \sup _{t \in \mathbf{R}}\left|w_{j+1}(t)\right| \quad \text { for } \quad 0 \leqq j<n
$$

Also we note $w_{n}(t)=f^{\prime}\left(q_{D}(y(t))\right) q_{D}(\dot{y}(t))$, and so using (H.1) we deduce there is a constant $N$ such that

$$
|\ddot{y}(t)| \leqq N \quad \text { for all } t \in \mathbb{R} \text {. }
$$

Lemma 4.3. Let $M>0$ and $P>0$. Suppose that $y(t)$ is a periodic solution of (4.4) with $|y(0)| \leqq M$ and with minimal period $p<P$. Then there exists a constant $R>0$ (independent of $D$ ) such that

$$
\left|y_{j}\left(t+\Delta_{j}\right)-y_{j+1}(t)\right| \leqq R \Delta_{j}^{2} \quad \text { for all } t \in \mathbb{R}, \quad 0 \leqq j<n .
$$

Proof. Let $t \in \mathbb{R}$. Then

$$
\begin{aligned}
y_{j}\left(t+\Delta_{j}\right)-y_{j+1}(t) & =y_{j}\left(t+\Delta_{j}\right)-y_{j}(t)+y_{j}(t)-y_{j+1}(t) \\
& =y_{j}\left(t+\Delta_{j}\right)-y_{j}(t)-\Delta_{j} \dot{y}_{j}(t)
\end{aligned}
$$

(since $y(t)$ satisfies (4.4)). By Taylor's Theorem there exists $\xi$ with $t<\xi<t+\Delta_{j}$ such that

$$
y_{j}\left(t+\Delta_{j}\right)-y_{j+1}(t)=\left(\Delta_{j}^{2} / 2\right) \ddot{y}_{j}(\xi) .
$$

The lemma follows from Lemma 4.2.

Remark 4.4. Let $y(t)$ satisfy the conditions of Lemma 4.3 and let $-r \leqq \theta \leqq 0$. Suppose $j$ is such that $t_{j} \leqq \theta<t_{j+1}$. Then

$$
\begin{aligned}
y_{j}(t)-y_{n}(t+\theta)= & y_{j}(t)-y_{j+1}\left(t-\Delta_{j}\right)+y_{j+1}\left(t-\Delta_{j}\right)-y_{j+2}\left(t-\Delta_{j}-\Delta_{j+1}\right) \\
& +y_{j+2}\left(t-\Delta_{j}-\Delta_{j+1}\right)-\cdots+y_{n}\left(t-\Delta_{j}-\Delta_{j+1}-\cdots-\Delta_{n-1}\right)-y_{n}(t+\theta) .
\end{aligned}
$$

But $\Delta_{j}+\Delta_{j+1}+\cdots+\Delta_{n-1}=t_{n}-t_{j}=-t_{j}$, so we have by Lemma 4.3

$$
\begin{aligned}
\left|y_{j}(t)-y_{n}(t+\theta)\right| & \leqq R\left(\Delta_{j}^{2}+\Delta_{j+1}^{2}+\cdots+\Delta_{n-1}^{2}\right)+\left|y_{n}\left(t+t_{j}\right)-y_{n}(t+\theta)\right| \\
& \leqq \Delta r R+\Delta L=(L+r R) \Delta
\end{aligned}
$$

where $L$ is the constant in Lemma 4.2. Thus there is a constant $Q>0$ (independent of $D$ ) 
such that

$$
\left\|y_{n, t}-q_{D}(y(t))\right\|<Q \Delta \text { for all } t \in \mathbb{R} \text {. }
$$

This inequality is used in the next theorem.

Now choose a sequence $\left\{D_{m}\right\}$ of dissections of $[-r, 0]$ with $\Delta_{D_{m}} \rightarrow 0$. Let $n_{m}$ be the number of intervals in $D_{m}$ and for convenience we write $\Delta_{m}$ for $\Delta_{D_{m}}, f_{m}$ for $f_{D_{m}}, Y_{m}$ for $\mathbb{R}^{n_{m}+1}, p_{m}$ for $p_{D_{m}}, q_{m}$ for $q_{D_{m}}$ and $T_{m}$ for the flow generated by $\dot{y}=f_{m}^{m}(y)$ in $Y_{m}$. It is clear that $\left\{Y_{m}, p_{m}, q_{m}\right\}$ is an approximation scheme for $X$ and by (4.2) and (4.3) we see that $T$ is approximable with respect to this approximation scheme. In fact we prove:

Theorem 4.5. Let $T$ be the $C^{0}$-semiflow generated by (4.1) in $X$. Then $T$ is $F$-proper with respect to the approximation scheme $\left\{Y_{m}, p_{m}, q_{m}\right\}$.

Proof. Let $\left\{m_{j}\right\}$ be a sequence of integers with $m_{j} \rightarrow \infty$ and suppose $\left\{\left(t_{m_{j}}, x_{m_{j}}\right)\right\}$ is a corresponding bounded sequence in $[0, \infty) \times X$ with $x_{m_{j}}=q_{m_{j}}\left(y_{m_{j}}\right)$ for some $y_{m_{j}} \in Y_{m_{j}},\left\{t_{m_{j}}\right\}$ bounded away from zero and such that $y_{m_{j}}=T_{m_{j}}\left(t_{m_{j}}, y_{m_{j}}\right)$. Put $\tilde{y}_{m_{j}}(t)=T_{m_{j}}\left(t, y_{m_{j}}\right)$ so $\tilde{y}_{m_{j}}(t)$ is a periodic solution of $\dot{y}=f_{m_{j}}(y)$ of period $t_{m_{j}}$. Since $\left\{x_{m_{j}}\right\}$ is bounded there exists $M>0$ such that $\left|\tilde{y}_{m_{j}}(0)\right| \leqq M$ and we may assume $t_{m_{j}} \rightarrow \tau>0$ (passing to a subsequence if necessary). Choose $P>\max \left\{t_{m_{j}}\right\}$. Consider the sequence $\left\{\tilde{y}_{m_{j}, n_{m}}\right\}$ of periodic functions from $\mathbb{R}$ to $\mathbb{R}$, we consider these functions as elements of $C([-r, P], \mathbb{R})$. By Lemma 4.1 and Lemma 4.2 and applying the Ascoli-Arzela Theorem, we may assume $\tilde{y}_{m_{j}, n_{m}} \rightarrow \tilde{x}$ in $C([-r, P], \mathbb{R})$. By $(4.5)$ we deduce that $q_{m_{j}}\left(\tilde{y}_{m_{j}}(t)\right) \rightarrow \tilde{x}_{t}$ in $X$ uniformly for $t \in[0, P]$. In particular $x_{m_{j}} \rightarrow \widetilde{X}_{0}$. Since

$$
\tilde{y}_{m_{j}, n_{m_{j}}}(t)=\tilde{y}_{m_{j}, n_{m_{j}}}(0)+\int_{0}^{t} f\left(q_{m_{j}}\left(\tilde{y}_{m_{j}}(s)\right)\right) d s
$$

$\left(\tilde{y}_{m_{j}}\right.$ is a solution of $\left.\dot{y}=f_{m_{j}}(y)\right)$ it follows that

$$
\tilde{x}(t)=\tilde{x}(0)+\int_{0}^{t} f\left(\tilde{x}_{s}\right) d s
$$

for all $0 \leqq t \leqq P$. Thus $\tilde{x}$ is a solution of (4.1). As $\tilde{y}_{m_{j}}\left(t_{j}\right)=\tilde{y}_{m_{j}}(0)$ and $t_{j} \rightarrow \tau>0$ it follows that $\tilde{x}_{0}=\tilde{x}_{r}$. Thus $\tilde{x}$ is a periodic solution of (4.1) with period $\tau$, that is $\tilde{x}_{0}=T\left(\tau, \tilde{x}_{0}\right)$. The theorem is proved.

This theorem shows that the generalised Fuller index can be used to study periodic solutions of functional differential equations. Although the generalised Fuller index is possibly many-valued, the techniques used in [2], [3], [4] still apply and similar results can be proved using the generalised index.

\section{Further remarks}

We have noted that the generalised Fuller index can be used to study functional differential equations (f.d.e.s). Also we believe that the generalised index can be used to 
study semiflows arising in other areas of mathematics, for instance non-linear partial differential equations. However our results in this direction are not well developed so we do not include them here.

It should be pointed out that Chow and Mallett-Paret have developed a Fuller index for f.d.e.s in [2]. But we suggest that the generalised index has an advantage, namely that difficult results concerning generic bifurcations for 1-parameter families of f.d.e.s are avoided. Perhaps more interestingly the techniques used in this paper provide an alternative approach to the Fuller index for f.d.e.s.

Consider the f.d.e.

$$
\dot{x}(t)=f\left(x_{t}\right) .
$$

Let $\Omega$ be a bounded open subset of $[0, \infty) \times X$, bounded away from $\{0\} \times X$ and let $T$ be the $C^{0}$-semiflow generated by (5.1) (we use the notation and hypotheses of Section 4). If $\Pi(T) \cap \partial \Omega=\phi$ then we have seen that $I(T, \Omega)$ is well defined, but possibly contains more than one element. Using the techniques of Section 4 it is possible to prove that there exists an $\varepsilon>0$ such that $i\left(f_{D}, \Omega_{D}\right)$ is defined for all dissections $D$ of $[-r, 0]$ with $\Delta_{D}<\varepsilon$. (Note that $\Omega_{D}=\left\{(t, y) \in[0, \infty) \times \mathbb{R}^{n(0)+1}:\left(t, q_{D}(y)\right) \in \Omega\right\}$.) In fact we claim that this index "stabilises".

Proposition 5.1. There exists an $\varepsilon>0$ such that if $D$ and $D^{\prime}$ are dissections of $[-r, 0]$ with $\Delta_{D}, \Delta_{D^{\prime}}<\varepsilon$ then

$$
i\left(f_{D}, \Omega_{D}\right)=i\left(f_{D^{\prime}}, \Omega_{D^{\prime}}\right)
$$

Thus the generalised index $I(T, \Omega)$ contains exactly one element. In fact we believe that this index is the same as Chow and Mallet-Paret's index though we have not, as yet, proved this. We hope to write up these results in a subsequent paper.

\section{REFERENCES}

1. F. E. Browder and W. V. Petryshyn, Approximation methods and the generalised topological degree for non-linear mappings in Banach space, J. Funct. Anal. 3 (1968), 217-245.

2. S. N. Chow and J. Mallet-Paret, The Fuller index and global Hopf bifurcation, $J$. Differential Equations 39 (1978), 66-84.

3. F. B. Fuller, An index of fixed point type for periodic orbits, Amer. J. Math. 89 (1967), 133-148.

4. R. D. Nussbaum and A. J. B. Potter, Cyclic differential equations and period three solutions of differential-delay equations, J. Differential Equations 46 (1982), 379-408.

5. A. J. B. Potter, On a generalisation of the Fuller index, Proceedings of the 1983 AMS Summer Institute on non-linear functional analysis and applications, to appear.

UNIVERSITY OF ABERDEEN

Aberdeen AB9 2TY

SCOTLAND 